\title{
Eine neue Disziplin formiert sich
}

\section{Zum Stand der Sozialinformatik in Deutschland}

Helmut Kreidenweis

Der Einsatz der Informationstechnologie (IT) hat Einzug auch in soziale Organisationen gehalten. Große Defizite gibt es jedoch nach wie vor beim IT-Einsatz für die Kernprozesse sozialer Hilfe - was auch an fehlenden fachlichen Standards der Sozialen Arbeit liegt.

Vor 25 Jahren erschien erstmals ein Themenheft der Blätter der Wohlfahrtspflege über den Einsatz der Computertechnik in der Sozialen Arbeit (Heft 8/1983); vor zehn Jahren gab es die erste Ausgabe speziell zum Thema Sozialinformatik (Heft 6/1997). "Technologisch unterbelichtet « sei die Soziale Arbeit, bemerkte darin Bernd Halfar, weil in einem vom Selbstkostendeckungsprinzip geschützten $\mathrm{Sy}$ stem das "potenzielle Konkursrisiko als notwendiges Korrektiv « fehle. Manches hat sich seither in der Sozialen Arbeit geändert. Doch wo steht die Sozialinformatik heute und was sind ihre künftigen Perspektiven?

Zunächst eine Rückblende ins Jahr 1997: Mit seinem programmatischen Statement »Sozialinformatik unerlässlich« (a. a. O.) war Halfar damals ein einsame Rufer in der Wüste. Zwar boten mehrere Hochschullehrer seit den 1980er Jahren Kurse für »EDV in der Sozialarbeit « an und trafen sich von Zeit zu Zeit in einer Arbeitsgruppe, doch es gab für dieses Fachgebiet noch keine einzige Professur. In der Praxis war Informationstechnologie längst ein Thema: Es existierten schon zahlreiche, primär verwaltungsorientierte Computerprogramme und ab Anfang der 1990er Jahre wurden spezielle Fachmessen veranstaltet, auf de-

Prof. Helmut Kreidenweis lehrt Sozialinformatik an der Katholischen Universität Eichstätt-Ingolstadt. Er ist zudem Inhaber der IT-Beratung KI Consult in Augsburg.

E-Mail

helmut.kreidenweis@ku-eichstaett.de nen sich Anbieter und Anwender trafen. Doch im harten Kern der Sozialen Arbeit galt die Kugelkopf-Schreibmaschine noch als Speerspitze der technologischen Revolution und der Gedanke an eine Disziplin, die fachliches Handeln systematisch mit Methoden der Informatik verknüpft, war neu.

Einen ersten Meilenstein in der disziplinären Entwicklung setzte Wolf Rainer Wendt im Jahr 2000 mit dem Sammelband "Sozialinformatik - Stand und Perspektiven «. Er verortete das neue Fachgebiet in doppelter Hinsicht: Zum einen definierte er seinen Gegenstandsbereich mit Sozialen Arbeit und Sozialwirtschaft, integrierte also neben der Fachlichkeit auch Aspekte des Managements und der Administration. Zum anderen bestimmte er seine Aufgaben mit "technischer Konzipierung, Ausführung und Evaluation « $(\mathrm{S}$. 20), welche die Prozesse der Anforderungsdefinition, der Einführung und Nutzung sowie deren wissenschaftliche Analyse umfassen. Damit erweiterte Wendt den Horizont: Er stellte die Sozialinformatik erstmals in den Kontext anderer Fachinformatiken und eröffnete ihr so eine Technik gestaltende und fachwissenschaftliche Perspektive. Wenn es auch zuvor schon Denkansätze in dieser Richtung gab (Kirchlechner 1987, 2000), so konzentrierten sich die "EDV-Pioniere" an den Hochschulen bis dato doch größtenteils auf die Aneignung vorhandener Technologien (»Computer für Sozialarbeiter «) und auf sozialwissenschaftlich geprägte Technologiekritik.

In den Folgejahren setzte langsam Fachdiskussion um die Sozialinformatik ein. Einig sind sich die Akteure bis heute weitgehend darin, dass sie als Disziplin primär in der Sozial(arbeits)wissenschaft und nicht in der Informatik beheimatet ist. Unterschiedliche Auffassungen gibt es teilweise zu Gegenstand und Aufgaben der Sozialinformatik. Jurgovsky (2004) kritisierte etwa, sie würde sich der Ebene der Programmierung verwei- gern und fordert eine »Sozioinformatik «, die eine interaktive Kulturtechnik für das Gemeinwesen darstellt und Programmierung als emanzipativen Prozess begreift. Ley (2004) sieht als Gegenstandsbereich die »Interaktionen des Sozialpädagogen « (S. 11) und grenzt damit betriebswirtschaftlich-administrative Aspekte aus.

Hinsichtlich des Aufgabenspektrums wird in erster Linie darüber gestritten, ob die Sozialinformatik auch den technischen Prozess der Programmierung umfasst. Dabei differenzieren die Diskutanten jedoch nur selten zwischen verschiedenen Ebenen der Ausbildung, Forschung und Entwicklung. Je nach Ebene kann die Antwort hier unterschiedlich ausfallen: Während im grundständigen, durch die Bachelor-Umstellung verkürzten Studium der Sozialen Arbeit ein Schnellkurs Programmierung weder Kompetenz- noch Profilgewinne zu generieren im Stande ist, wären Datenmodellierung oder Programm-Entwicklung in einem speziellen Sozialinformatik-Masterstudiengang vermutlich wichtige Elemente. Ähnliches gilt etwa für die Entwicklung neuartiger Software-Prototypen im Rahmen interdisziplinär angelegter Forschungs- und Entwicklungsprojekte. Aufgabe einer wissenschaftlichen Disziplin ist es jedoch nirgends, Lösungen der Informationstechnologie (IT) für die Praxis auszuentwickeln und dauerhaft Wartung und Support dafür bereitzustellen.

Bei aller notwendigen Kritik an der aktuellen Ausrichtung der Sozialinformatik sollte bedacht werden, dass dieser Begriff bislang eher als Ticket auf dem Weg zu einer Disziplin denn als Zielbahnhof gesehen werden muss.

Wo steht aber die Sozialinformatik heute? Auf der Suche nach Antworten gilt es zwischen dem Stand der IT-Entwikklung, dem IT-Einsatz in der Praxis sowie der Lehre und Forschung zu differenzieren. Statt eines umfassenden Überblicks, folgen einige Schlaglichter. 


\section{Stand der Fachsoftware- Entwicklung: Es fehlen spezifische Programme - weil fachliche Standards fehlen}

Fachspezifische Software, ihre Konzipierung, Implementation, Anwendung und die wissenschaftliche Reflexion dieser Prozesse spielt - wie in allen Fachinformatiken - auch in der Sozialinformatik eine tragende Rolle. Im eingangs erwähnten Themenheft dieser Zeitschrift zur Sozialinformatik schrieb der Autor diese Beitrages dazu vor zehn Jahren: »EDV im Sozialwesen bedeutet in absehbarer Zukunft meist nichts anderes, als Daten mehr oder minder mühsam zu erfassen und sie einzeln oder auf verschiedene Weise kumuliert und verknüpft wieder abzurufen. « (S. 117). Daran hat sich bis heute nichts geändert. Ist die IT-Entwicklung im Sozialbereich also seither stehen geblieben? Keineswegs, doch Quantensprünge blieben bislang aus. Verflogen ist die frühe Euphorie mancher Computer-Propheten ebenso wie die paramilitärische Bekämpfung alles Digitalen. Stattdessen sind Pragmatismus und eine langsame, bisweilen zähe Entwikklung hin zu professionelleren Lösungen eingekehrt.

Neben einer Ausdifferenzierung betriebswirtschaftlicher Funktionen gewann die Abbildung fachlicher Verfahren mehr und mehr an Bedeutung. Häufig steht dabei die Schnittstelle zwischen Fachlichkeit und Administration im Vordergrund: Hilfebedarfsplanung, Leistungserfassung und Berichtswesen sind typische Beispiele. Allmählich rückt auch die IT-gestützte Bearbeitung unmittelbar hilferelevanter Informationen in den Blickpunkt des Interesses. Doch die meisten Programme sind noch nicht entlang der tatsächlichen Arbeitsprozesse der Praxis konzipiert, eine durchgängige IT-Unterstützung in Kernprozessen wie Diagnostik, Hilfeplanung, Dokumentation und Evaluation ist noch selten. Auch basieren nicht wenige Lösungen noch auf Technologien der 1980er und 1990er Jahre. Zur Suche von Software, die sich an modernen Standards flexibler Systemarchitekturen, Datenmodellen und Benutzeroberflächen orientiert, benötigt man eine Lupe.

"Ausgangspunkt der Softwarekonstruktion sollte das fachliche Problem sein «, so Halfar im Jahre 1997. Sozialarbeit brauche die "Definitionsmacht in der Software-Konstruktion « (S. 113). Davon ist die Realität auch heute noch ein gutes Stück entfernt. Software wird noch immer aus einer diffusen Gemengelage wirtschaftlicher und fachlicher Anforderungen der Träger, einzelner Versatzstücke diagnostischer und analytischer Verfahren sowie Eigenideen der Herstellerfirmen entwickelt. Die Fachlichkeit ist dabei vielfach durch Sachzwänge, Traditionen oder überkommene Wissensstände gebrochen. Nach wir vor stellt sich die von Mosebach/Göppner im Jahr 2005 aufgeworfene Frage: Ist Sozialarbeit drin, wo Sozialarbeit drauf steht?

Die Analyse aktuelle angebotener Programme fördert ernüchternde Ergebnisse zu Tage. Da werden Fachterminologien bunt durcheinandergewürfelt, Unmengen an defizitorientierten Klientendaten abgefragt oder wichtige Elemente wie personale oder Umfeld-Ressourcen bleiben ausgeblendet. »Dafür haben Sie ja dann das Freitextfeld links unten «, lautet nicht selten die Antwort der Hersteller auf entsprechende Fragen. Andererseits: Wie sollten sie gute Software produzieren und

\section{Zitat}

»Die Idee der Bürgergesellschaft wäre gescheitert, käme sie über bloBes staatsergänzendes und staatsentlastendes Handeln nicht hinaus. Denn sie ist mehr als die Addition von Spendensummen, ehrenamtlicher Arbeit und gemeinnützigen Organisationen. Analog zum marktlichen Steuerungsideal der Konsumentensouveränität, wäre die Orientierung an einer Bürgersouveränität als letztgültigem » Regler « gesellschaftlicher Prozesse, das Steuerungsideal für eine auf Selbstorganisation basierende Bürgergesellschaft."

Dr. Stefan Nährlich, Geschäftsführer Aktive Bürgerschaft e. V., in den Blättern der Wohlfahrtspflege 4/2007

verkaufen, wenn, wie etwa vielfach in der Jugendhilfe, anerkannte prozessuale und inhaltliche Standards fehlen und die Einrichtungen auf höchst unterschiedlichen fachlichen und organisatorischen Niveaus agieren?

Eine systematische Entwicklung von Software auf dem Hintergrund wissenschaftlich fundierter Fachkonzepten wie Case-Management oder auf Basis theoretisch stringenter Diagnose-, Interventions- oder Partizipationsmodellen findet noch kaum statt. Die mittlerweile häufig anzutreffenden Versuche, aus der Forschung heraus entwickelte Diagnostikoder Ablauf-Schemata an die vorhandene Software anzukleben, laufen ins Leere, wenn weder Anbieter noch Anwender deren theoretischen oder methodischen Bezugsrahmen in ihr Gesamtkonzept integrieren.

\section{Informationstechnologie in der Praxis: Der Wechsel vom Fax zum E-Mail ist geschafft}

Die Formen der Nutzung von Informationstechnologie in der Praxis Sozialer Arbeit sind so vielfältig, wie die Soziale Arbeit selbst, ein einigermaßen umfassender Überblick ist kaum möglich. Der von Halfar 1997 noch vermutete »cultural lag größeren Ausmaßes « (S. 114) in Sachen Informationstechnologie und Management sozialer Organisationen kann heute für die Mehrzahl der großen Träger als überwunden gelten. Doch viele Führungskräfte mittlerer oder kleinerer Einrichtungen sehen die Computertechnik noch weit eher als lästigen Kostenblock denn als strategischen Erfolgsfaktor. Auch nicht wenige Praktiker verspüren Unbehagen bei der Einführung von ITLösungen in ihrem Arbeitsbereich. An die Stelle der früheren Technikangst ist heute die Furcht vor Ökonomisierung und Konformisierung Sozialer Arbeit getreten, die intuitiv mit dem Einsatz von Fachsoftware in Zusammenhang gebracht wird.

Dennoch haben sich PCs, Office- und Kommunikationssoftware längst überall etabliert. Von einer wirklich effizienten IT-Nutung sind viele Sozialorganisationen aber noch Lichtjahre entfernt. Da basteln Sozialarbeiter tagelang an Excel-Tabellen für Aufgaben, die mit preisgünstiger Fachsoftware im Handumdrehen zu 
lösen wären. Schlecht geschulte Sekretärinnen treten Lawinen von Notlösungen los, weil sie ihre Programme nicht kennen und Führungskräfte verkünden stolz, sie würden »Informationsmanagement « betreiben, wenn Sie ihre Mitarbeiter täglich mit E-Mails überhäufen. Informationstechnologie wird vielfach noch nicht als das begriffen, was sie ist: eine Management-Aufgabe, die einer angemessenen personellen und organisatorischer Verankerung im Unternehmen bedarf. Freilich, es gibt viele positive Beispiele: Sozialarbeiter berichten von einer verbesserter Strukturierung ihrer Arbeit (z. B. Kreidenweis, 2005) oder neuartige Wissensmanagement-Systeme stiften Nutzen und finden breite Akzeptanz (Fexer u. a. 2005).

Wenig Fortschritte gibt es bislang in der trägerübergreifenden Interaktion, deutlichere Spuren als der Wechsel vom Fax zur (meist völlig ungesicherten) E-Mail hat die technologische Revolution hier noch nicht hinterlassen. Interaktive Datenbanksysteme, die freie Plätze anzeigen, ein standardisiertes elektronisches Berichtswesen oder auch nur die papierlose Übermittlung der Leistungsabrechnung sind seltene Leuchttürme. Lediglich in neueren Anwendungsgebieten wie dem Benchmarking haben sich internetbasierte Lösungen zum Betriebsvergleich etabliert.

Ein großes Entwicklungsgebiet stellt nach wie vor die Nutzung telematischer Lösungen, etwa in der ambulanten Betreuung dementer oder geistig behinderter Menschen dar. Hier sollte die Soziale Arbeit das Feld nicht länger allein anderen Akteuren wie Ingenieurwissenschaftlern oder Informatikern überlassen.

\section{Lehre und Forschung: Nach Jahren des Stillstands kommt Bewegung in die Szene}

An den Hochschulen kommt die Etablierung der Sozialinformatik nur zögernd voran. Eine auf ein Einzelsemester bezogene Internet-Recherche im Jahr 2006 ergab, dass nur etwa ein Fünftel aller Ausbildungsstätten für Soziale Arbeit Veranstaltungen aus diesem Themenfeld anboten (Janatzek 2007, S. 55). Aktuell gibt es an acht Prozent der entsprechenden Hochschulen eigene Professuren für das Fach (Voll- oder Teilzeit) und an einigen weiteren Kombinationen mit anderen Fachgebieten. In manchen Masterstu- diengängen für Sozialmanagement sind entsprechende Module verankert, einen speziellen Sozialinformatik-Master gibt es in der Schweiz (FHS St. Gallen), ein erster deutscher Weiterbildungsstudiengang wird derzeit an der Katholischen Universität Eichstätt-Ingolstadt geplant. schen Themen statt. Als jährliche Veranstaltung beginnt sich seit 2006 die Eichstätter Sozialinformatik-Tagung zu etablieren. Die kürzlich gegründete Arbeitsstelle für Sozialinformatik an der Katholischen Universität Eichstätt-Ingolstadt gibt ab Herbst 2007 einen perio-

\section{»Viele Programme fragen einfach eine Unmenge defizitorientierter Klientendaten ab«}

Nach Jahren der Stille seit Auflösung der erwähnten Hochschullehrer-AG kommt mittlerweile auch in den wissenschaftlichen Dialog wieder Bewegung. Dieser geht jedoch heute über die Hochschulgrenzen hinaus und bezieht Anwender und Anbieter der IT-Lösungen mit ein. Im Jahre 2005 gab es zwei Tagungen zur Lehre und Forschung in der Sozialinformatik (Kreidenweis/Ley 2006) und im Jahr 2007 fanden nach Kenntnis des Autors deutschlandweit immerhin vier Fachtagungen $\mathrm{zu}$ dezidiert sozialinformati- disch erscheinenden IT-Report für die Sozialwirtschaft heraus, der erstmals empirisch fundierte Informationen zum Einsatz von Informationstechnologie in sozialen Organisationen und zum Angebot an Fachsoftware liefert. Vermehrt werden Forschungsthemen im Rahmen von Abschlussarbeiten und Dissertationen bearbeitet, eine erste Materialiensammlung ist bereits im Internet verfügbar (http://www.sozialinformatik.de).

Die bisher vorherrschenden Wege der Software-Entwicklung und deren Ergeb-

\section{Literatur}

Fexer, Helmar; Kreidenweis, Helmut; Kurz, Andreas: IT-gestütztes Wissensmanagement. Teures Spielzeug oder wertvolles Instrumentarium? - Ergebnisse eines Pilotprojektes. In: Nachrichtendienst des Deutschen Vereins, Nr. 11/2005: S. 432-435.

Mosebach, Ursula; Göppner, Hans-Jürgen: Sozialinformatik. Studieren in virtuellen Räumen. In: Sozialmagazin, $\mathrm{Nr}$. 6/2005: S. 46-53.

Halfar, Bernd: Sozialinformatik unerlässlich. In: Blätter der Wohlfahrtspflege, Nr. 6/1997: S. 113-114.

Janatzek, Uwe: Sozialinformatik in der Sozialen Arbeit. Saarbrücken, 2007.

Jurgovsky, Manfred: Sozioinformatik. Ein Vorschlag zur Neupositionierung der Informatik in der Sozialen Arbeit. In: Archiv für Wissenschaft und Praxis der sozialen Arbeit, Nr. 1/2004: S. 4048.

Kirchlechner, Berndt: Computereinsatz in Lehre, Forschung und Organisation an den Fachbereichen Sozialpädagogik und Sozialarbeit der Fachhochschule Frankfurt/Main. In: Frommann, Mat- thias (Hg.):Dezentrale Elektronische Datenverarbeitung in der sozialen Arbeit, Frankfurt am Main, 1987.

Kirchlechner, Berndt: Die AG Computer und Sozialarbeit. In: Wendt, Wolf Rainer (Hg.): Sozialinformatik: Stand und Perspektiven, Baden-Baden, 2000.

Kreidenweis, Helmut: Software für die Sozialarbeit. Aktuelle Trends und nüchterne Analysen. In: Blätter der Wohlfahrtspflege, Nr. 6/1997: S. 115-117. Kreidenweis, Helmut: IT-gestützte Hilfeplanung im Jugendamt. Wie wirkt sich Fachsoftware auf den Planungsprozess aus? In: Nachrichtendienst des Deutschen Vereins für öffentliche und private Fürsorge, Nr. 6/2005: S. 196203.

Kreidenweis, Helmut; Ley, Thomas (Hg.): Sozialinformatik in Lehre und Forschung - Von der Standortbestimmung zur Zukunftsperspektive. (Elektronische Publikation, www.sozialinformatik.de) Eichstätt/Bielefeld, 2006.

Wendt, Wolf Rainer (Hg.): Sozialinformatik. Baden-Baden, 2000. 
nisse zeigen die Notwendigkeit zur stärkeren Verzahnung von Programmierung, sozialarbeiterischer Praxis, Methodenlehre und Sozialinformatik. Eine wichtige Voraussetzung dafür ist die Intensivierung des Dialoges zwischen Sozialarbeitswissenschaft, Sozialmanagement-Theorie und Sozialinformatik: Noch immer wird in der Literatur zu Fach- und Führungspraxis meist so getan, als wäre der Computer nur eine Schreibmaschine ohne Tipp-Ex. Hier gilt es, die sozialinformatischen Optionen bereits bei der Entwikklung und Implementierung neuer Methoden und Handlungsansätze systematisch zu integrieren. Als Modell können etwa Wirtschaftswissenschaft und Wirtschaftsinformatik dienen, in der Konzepte wie das Geschäftsprozess- oder Informationsmanagement betriebswirtschaftliche und informatische Elemente nahtlos verschmelzen.

\section{Weitere Aussichten: heiter bis wolkig}

Ein letzter Sprung zurück ins Jahr 1997: Der von Halfar damals geforderte »informationstechnische Quantensprung « im Feld der Sozialen Arbeit ist ausgeblieben. Ein von den Kernprozessen Sozialer Arbeit ausgehendes Informationsmanagement in sozialen Einrichtungen ist noch immer mehr Vision als Realität. Stattdessen bewegte sich die Soziale Arbeit in langsamen Tippelschritten in Richtung einer besseren Nutzung der Technik für ihre Aufgaben. Als Hindernisse werden immer wieder fehlende inhaltliche und prozessuale Standards genannt, die tieferen Gründe sind jedoch in der mangelnden Integration informatischen Denkens in die gängigen Organisations- und Handlungsmodelle zu suchen. Nicht zuletzt spielt auch das fehlende Geld - oder besser - die mangelnde Investitionsbereitschaft eine Rolle.

Doch der Beweis dafür, dass Informationstechnologie den Outcome sozialer Organisationen verbessert, ist bis heute nicht erbracht. Noch weniger wissen wir über die Kosten-Nutzen-Relation oder gar über Grenzkosten und Grenznutzen bei der Technik-Einführung: Bringt mehr Funktionalität auch höheren Nutzen? Oder gibt es einen Ausstattungsgrad, von dem Aufwärts kein Zusatznutzen mehr $\mathrm{zu}$ erwarten ist? Ungeklärt ist auch, ob und gegebenenfalls wo Grenzen bei der IT-Durchdringung von Aufgabenfeldern Sozialer Arbeit zu ziehen sind: Zeitigt eine offene, papiergestützte Form der Hilfeplanung gegenüber einer stark durch Software vorstrukturierten bessere oder schlechtere Ergebnisse für Kostenträger und Adressaten?

Wichtigste Aufgabe der Sozialinformatik sollte es in den nächsten Jahren sein, die empirische Forschung voranzutreiben und mit der Sozialarbeits- und Sozialwirtschaftsforschung zu vernetzen. Erkenntnisse der Forschung werden gleichermaßen für die Weiterentwicklung der Praxis wie als Basis für die Theoriebildung gebraucht. Mit Hilfe grundlagenorientierter und anwendungsbezogener Forschungssettings kann mehr Klarheit über Wirkungen, nicht intendierte Nebenwirkungen und Rahmenbedingungen des IT-Einsatzes in sozialen Organisationen und im Adressatendialog geschaffen werden. Auf ihrer Basis können etwa evidenzbasierte Handreichungen für eine optimale Techniknutzung in Fachlichkeit und Management entwickelt werden.

Bislang haben freilich die klassischen Forschungsförderer der Branche das Gestaltungspotenzial durch Informationstechnologien in der Sozialen Arbeit noch nicht erkannt. Auch die Spitzenebenen Wohlfahrtsverbände betrachten die ITAnwendung meist noch als technisches Problem ihrer Untergliederungen. Ihre sozialpolitische Dimension, etwa zum Nachweis der Wirksamkeit ihrer Arbeit oder zur Vernetzung von Sozial- und Gesundheitsdiensten, muss erst noch in das Blickfeld der Funktionäre gerückt werden.

Als Disziplin muss die Sozialinformatik weiter ihren Weg zwischen Sozialer Arbeit, Sozialmanagement, Sozialarbeitswissenschaft und Angewandter Informatik suchen. Schon die aufgeworfenen Forschungsfragen zeigen, dass Fachlichkeit und Ökonomie dabei nicht zu trennen sind. Auch die Software-Entwicklung kennt keine akademischen Grenzziehungen: in modernen Systemen werden fachliche, verwaltungstechnische und Management-Funktionen auf immer höheren Niveaus integriert. Die Sozialinformatik wird also auch künftig die Spannung zwischen Ökonomie und Fachlichkeit nicht nur aushalten, sondern für die Weiterentwicklung ihrer Erkenntnisse und Methoden produktiv nutzen müssen.

\section{"Einer der besten seiner Zunft."}

Prof. Dr. H.-D. Gottlieb, Sozialrecht aktuell 1/99, zur Vorauflage

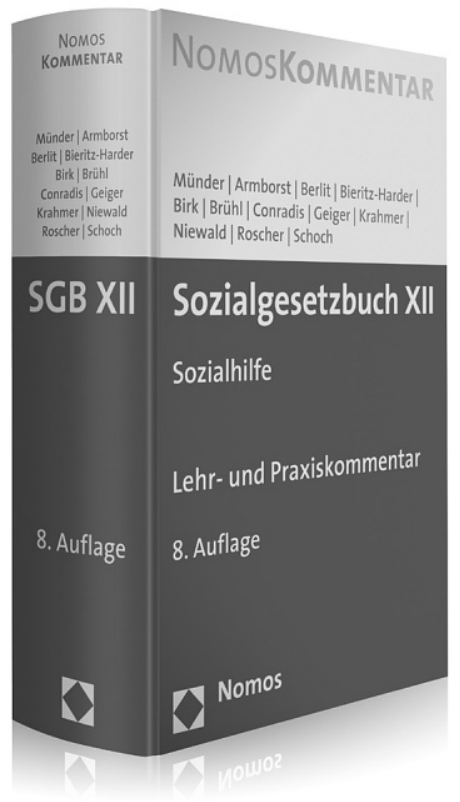

Sozialgesetzbuch XII Sozialhilfe

Lehr- und Praxiskommentar Von Prof. Dr. Johannes Münder u.a. 8. Auflage 2008, ca. 900 S., geb., ca. 44,- , ISBN 978-3-8329-2930-5 Erscheint Januar 2008

Die Neuauflage des LPK-SGB XII bringt Sie im Sozialhilferecht wieder auf den neuesten Stand.

"Eine unentbehrliche

Arbeitshilfe. Wohl kein anderer Kommentar kann einen vergleichbaren gebündelten Sachverstand an Autoren aus Universität, Gerichten und der Anwaltschaft aufbieten ". Rechtsdienst der Lebenshilfe 3/05, zur Vorauflage

\section{Nomos}

Bitte bestellen Sie bei Ihrer Buchhandlung oder bei Nomos | Telefon 07221/2104-37 | Fax -43 | www.nomos.de | sabine.horn@nomos.de 